\title{
ELECTROCATALYTIC OXIDATION OF TRYPTOPHAN AND TYROSINE USING DENSELY PACKED COBALT OXIDE SANDWICHED GRAPHENE STACKS MODIFIED ELECTRODE
}

\author{
R. Vivekananth ${ }^{1}$, V. Sivasankar ${ }^{1}$, E. Senthil Kumar ${ }^{1}$ and R. A. Kalaivani ${ }^{1, *}$ \\ ${ }^{1}$ Department of Chemistry, School of Basic Sciences, Vels University, Pallavaram, \\ Chennai 600 117, Tamil Nadu, India \\ *E-mail: rakvani@yahoo.co.in
}

\begin{abstract}
Herein, we construct a novel non-enzymatic, simple, selective and sensitive determination of Tryptophan and Tyrosine sensor. Cobalt oxide sandwiched graphene nanocomposite (COSG-NC) was successively synthesized through self-assembly method and immobilized on the surface of the wax impregnated graphite electrode via a simple drop and dry method. Surface topography of the densely packed COSG-NC electrode was confirmed by atomic force microscope analysis. The fabricated modified electrode showed an exceptional electrocatalytic response for Trp and Tyr oxidation occurred at a reduced overvoltage. Under optimal experimental conditions, the COSG-NC modified electrode demonstrated the extensive determination range from $24 \times 10^{-6} \mathrm{M}$ to $1 \times 10^{-3} \mathrm{M}$ and $41 \times 10^{-6} \mathrm{M}$ to $1 \times 10^{-6} \mathrm{M}$ with low limit of detection in the range of $8.2 \times 10^{-6} \mathrm{M}$ and $13 \times 10^{-6} \mathrm{M}(\mathrm{S} / \mathrm{N}=3)$ of $\operatorname{Trp}$ and Tyr, respectively. The superior responses of the modified electrode for Trp and Tyr have mainly ascribed to the nanocomposites having large surface area with more electroactive sites of $\mathrm{Co}_{3} \mathrm{O}_{4}$ and synergistic effect of graphene nanocomposites. This work offered a reasonable approach for future research in enzyme-free amperometric sensors for biomolecules determination.
\end{abstract}

Keywords: Cyclic Voltammetry, Tryptophan, Tyrosine, Graphene, Cobalt Oxide Nanoparticles

@) RASĀYAN. All rights reserved

\section{INTRODUCTION}

The expansion of effortless, selective, sensitive and authentic method for detecting amino acids is essential in many sectors, such as food industry, public health and ecological security. Several amino acids, for example, Tryptophan (Trp) and Tyrosine (Tyr), play essential responsibilities in the human system and living organisms for essential constituents of protein biosynthesis. Trp is the most significant amino acids for nitrogen balance in adults and average growth in children. It is a necessary precursor of the hormone for melatonin, serotonin, niacin, and other significant biomolecules. ${ }^{1,2}$ While, in the human body Trp cannot be synthesized directly, it can only be obtained through supplementations or foods. In overdoses, Trp may show side effects such as diarrhea, confusion, agitation, nausea, fever and so on. ${ }^{3}$ Tyr is also a type of necessary amino acid and essential for human to maintain nutritional balance. ${ }^{2,4}$ The deficiency of Tyr may lead to alkaptonuria, depression, albinism, and other psychological diseases, while an exceed of Tyr could cause the increase of sister chromatid exchange and dementia or Parkinson's diseases. ${ }^{5,6}$ Hence, it is important to develop an easy, precise, fast and low-cost technique for the determination of the small concentration of Trp and Tyr.

Recently, electrochemical methods have been the smartest technique for the determination of Trp and Tyr due to their advantageous properties such as simple operation, high sensitivity, precision, inexpensive and fast examination. Additionally, much interest has been directed in the detection of Tyr or Trp by electrochemical techniques using different modified electrodes. Since Trp often coexists with Tyr in pharmaceutical formulations, food processing, and biological fluids, different strategies have been developed for selective determination of Trp in the presence of Tyr. ${ }^{7,8}$ Hence, determination of Trp in the

Rasayan J. Chem., 12(3), 1166-1174(2019)

http://dx.doi.org/10.31788/RJC.2019.1235045

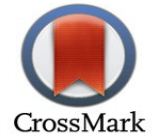


presence of Tyr with a good selectivity is a great challenge. P. Deng and coworkers fabricated a carbon paste electrode modified with graphene for selective detection of Trp in the presence of huge concentrations of Tyr. ${ }^{9}$ Ozcan and Sahin reported a different approach for the detection of Trp based on the electrochemical reduction of an oxidation product of Trp formed. ${ }^{10}$

Numerous nanomaterials based tailored electrodes have been reported for developing an electrochemical sensor for Trp and Tyr detection. ${ }^{11-16}$ Especially, the low-cost metal oxide nanoparticles, for instance, $\mathrm{CuO},{ }^{17,18} \mathrm{NiO}^{19}$ and $\mathrm{Co}_{3} \mathrm{O}_{4}{ }^{20-23}$ attract further interest owing to their inexpensive, size, shape, composition, crystallinity, structure, large surface area, excellent electrocatalytic activity and the opportunity of enhancing electron transfer reactions at a lesser potential of biomolecules determination. Among them, $\mathrm{Co}_{3} \mathrm{O}_{4}$ has acknowledged much attention owing to its good electrocatalytic ability and excellent chemical stability. Various $\mathrm{Co}_{3} \mathrm{O}_{4}$ nanomaterials-based amperometric sensors have also been prepared and useful for biomolecules sensing with good sensitivity, selectivity and stability. ${ }^{24-26}$ Graphene (GR) has recently immense attention because of its unique mechanical and electrical properties (e.g. fast electron transport property and good electrocatalytic activity). ${ }^{27,28} \mathrm{GR}$, the single layer of carbon atoms organized in a twodimensional honey-combed lattice in a flat structure and inexpensive nanomaterials, has been employed for developing devices of optoelectronics, field-effect transistors, supercapacitors, gas sensors, chemical sensors and biosensors. ${ }^{29-34}$

Herein, we exploit the advantages of $\mathrm{Co}_{3} \mathrm{O}_{4}$ and graphene; and we have constructed the selective electrooxidation of Trp and Tyr towards the successful fabrication of self-assembled COSG-NC coated on wax impregnated graphite electrode by drop and dry method. The COSG-NC modified electrode was usefully engaged for the detection of analytes using cyclic voltammetry, hydrodynamic voltammetry and chronoamperometric techniques. The obtained results indicated that the COSG-NC could be used as an enhanced electrocatalytic, selective and sensitive determination of Trp and Tyr.

\section{Instrumentation}

\section{EXPERIMENTAL}

Atomic force microscopy (AFM) was used to measure the surface topography of the COSG-NC modified electrode (Nanosurf EasyScan 2, Switzerland). Voltammetric measurements were executed by electrochemical workstation of $\mathrm{CHI} 760 \mathrm{D}$ ( $\mathrm{CH}$ Instruments, USA) connected by a computer. The electrochemical cell was constructed with a conventional 3-electrode system; a saturated calomel electrode (SCE) and a platinum wire were used as a reference and auxiliary electrode, respectively. The cobalt oxide sandwiched graphene nanocomposite (COSG-NC) modified electrode was used as the working electrode, in accordance with the synthesis procedure illustrated below. For amperometric measurements, a magnetic Teflon stirrer was presented for the convective mass transport. All experiments were executed at ambient temperature.

\section{Synthesis of the COSG-NC}

The synthesis of the COSG-NC was carried out using our earlier report ${ }^{22}$. Briefly, for the preparation of graphene amine (GR-A), initially graphene oxide (GO) was separately dispersed in water by ultrasonicator for an hour. Then, an aqueous solution of hexamethylene diamine (0.01 mole) is gradually added into graphene oxide solution and continuously stirred for 2 days. The obtained precipitate was centrifuged and three times washed with anhydrous ethanol and finally dried in a vacuum oven for 1 day. After that, in another beaker GO was dispersed in aqueous solution $(100 \mathrm{~mL})$ using ultrasonicator. An aqueous solution of $\mathrm{CoCl}_{2}(0.20 \mathrm{M})$ was slowly added dropwise into the $\mathrm{GO}$ solution and was stirred for $15 \mathrm{~min}$. GR-A (50 mg) was dispersed to the mixed solution containing ethanol and water (1:1). Finally, GR-A is slowly added to the $\mathrm{GO} / \mathrm{CoCl}_{2}$ solution and stirred for $15 \mathrm{~min}$, and 1 equivalent of $\mathrm{NaOH}$ was slowly added to the above solution until the $\mathrm{pH}$ reaches 12 . Finally, an aqueous solution of $(1 \mathrm{wt} \%) \mathrm{H}_{2} \mathrm{O}_{2}$ was added dropwise to the above mixture. Then the solution was heated at $120^{\circ} \mathrm{C}$ for $16 \mathrm{~h}$. Then, the obtained precipitate was washed with anhydrous ethanol and dried overnight in a conventional oven. The COSG-NC was pulverized and kept in a tube furnace in a flow of $\mathrm{Ar}$ gas and calcined at $400{ }^{\circ} \mathrm{C}$ for 120 $\min$. 


\section{Preparation of the COSG-NC Modified Graphite Electrode}

In our previous report, the construction of COSG-NC modified graphite electrode has been demonstrated. ${ }^{22}$ Briefly, impregnation of graphite electrode was done by a paraffin wax and then warm up the graphite rods in wax and connected with the vacuum suction for few minutes, till air bubbles stopped to evolve from the graphite rods. ${ }^{35}$ The graphite rods were withdrawn from paraffin wax before it solidified. The one end of the electrode surface was polished using various grades of emery sheets, thus obtaining with a fine mirror polish for surface modification of the electrode. Methanol and distilled water were used to wash the polished electrode surface. Later, one mg of COSG-NC was dispersed in $1 \mathrm{~mL}$ of $1 \%$ Nafion/ethanol solution and sonicated for 30 minutes. Ten microliters of the suspension were dropcast on the surface of wax impregnated graphite electrode and dried in ambient temperature.

\section{Atomic Force Microscope}

\section{RESULTS AND DISCUSSION}

The dimension and morphology of the COSG-NC were characterized by FESEM, XRD and XPS, which were reported in our earlier report. ${ }^{22}$ The surface topographic studies were carried out using AFM studies. For characterization purpose, COSG-NC was coated on the indium tin oxide (ITO) glass substrate and analyzed. Figure-1 shows the AFM topographic 3D images of the bare ITO and COSG-NC. The surface topography images evidently demonstrated the densely aligned graphene and cobalt oxide in the nanocomposite and the surface roughness root mean square (RMS) increased from the bare electrode surface. The surface roughness of bare ITO is $6.2 \mathrm{~nm}$ and the COSG-NC is $968.0 \mathrm{~nm}$. These results confirm the modification of the electrode surface with COSG-NC. AFM surface analysis discloses the analogous results of COSG-NC modified electrodes as that already acquired from the FESEM results. ${ }^{22}$
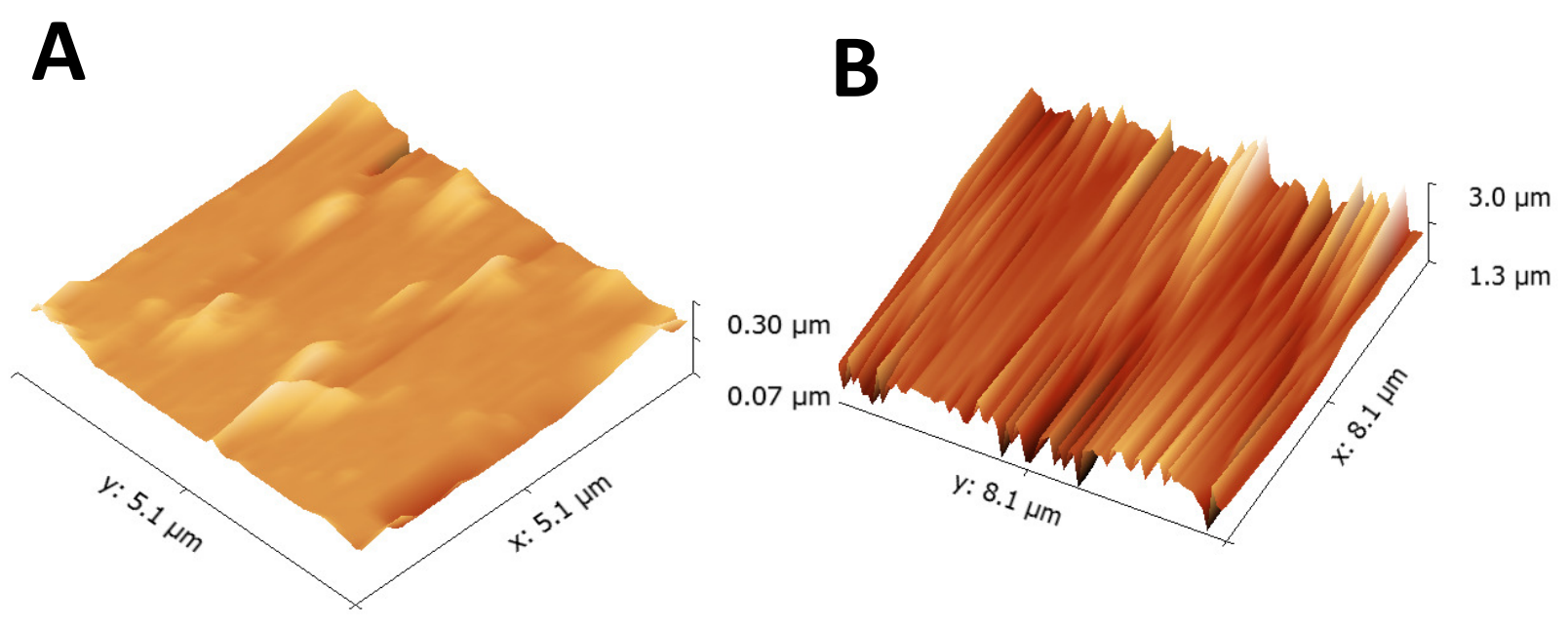

Fig.-1: AFM of the (A) bare ITO electrode and (B) COSG-NC Modified ITO Electrode.

\section{Electrocatalytic Oxidation of Trp and Tyr by Cyclic Voltammetry}

Figure-2A and B show the electrocatalytic behavior of Trp and Tyr at the COSG-NC modified graphite electrode and bare electrode in $0.1 \mathrm{M} \mathrm{NaOH}$. From curve b in Fig.-2, no considerable response was observed at the bare electrode for the electrochemical oxidation of Trp or Tyr. Hence, the bare electrode cannot be exploited for analytical detection of Trp and Tyr since it does not show any measurable current. However, in COSG-NC modified electrode, a sharp increase in the oxidation peak current was observed upon addition of Trp and Tyr and the cathodic current tends to decrease (curve d). In both cases, the oxidation peak currents increase with increasing Trp and Try concentration. The increment in the oxidation peak current discloses the efficient electrocatalytic behavior of the mediator. The improvement in oxidation peak current at the COSG-NC modified graphite electrode is ascribed to the oxidation of Trp and Tyr by $\mathrm{Co}^{\mathrm{III}}$ of cobalt oxide nanoparticles immobilized on the electrode surface during the anodic 
reaction. When the modified electrode is used, there is a reduction in overvoltage (about $100 \mathrm{mV}$ ) for the oxidation of Trp and Tyr compared to the bare electrode where the oxidation takes place above $0.45 \mathrm{~V}$ for Trp and $0.55 \mathrm{~V}$ for Tyr determination with the negligible current. The COSG-NC modified electrode exhibited the extensive linear range between $24 \mu \mathrm{M}-1 \mathrm{mM}$ and $41 \mu \mathrm{M}-1 \mathrm{mM}$ with a low detection limit of $8.2 \mu \mathrm{M}$ and $13 \mu \mathrm{M}(\mathrm{S} / \mathrm{N}=3)$ of Trp and Tyr, respectively. This demonstrates that COSG-NC at the modified electrode acts as an excellent electrocatalyst for determination of Trp and Tyr. Scheme-1 presents the mechanism of Trp and Tyr electrocatalytic oxidation at the COSG-NC modified electrode.

\section{Hydrodynamic Voltammetry Studies of Trp and Tyr}

The potential dependence of the amperometric response of the COSG-NC modified electrode was optimized with hydrodynamic voltammetry. The hydrodynamic voltammetry (HDV) measurements of various concentrations of Trp and Tyr for the COSG-NC modified electrode were carried out in the applied potential range of $0 \mathrm{~V}$ to $0.6 \mathrm{~V}$ as shown in Fig.-3A and 4A.
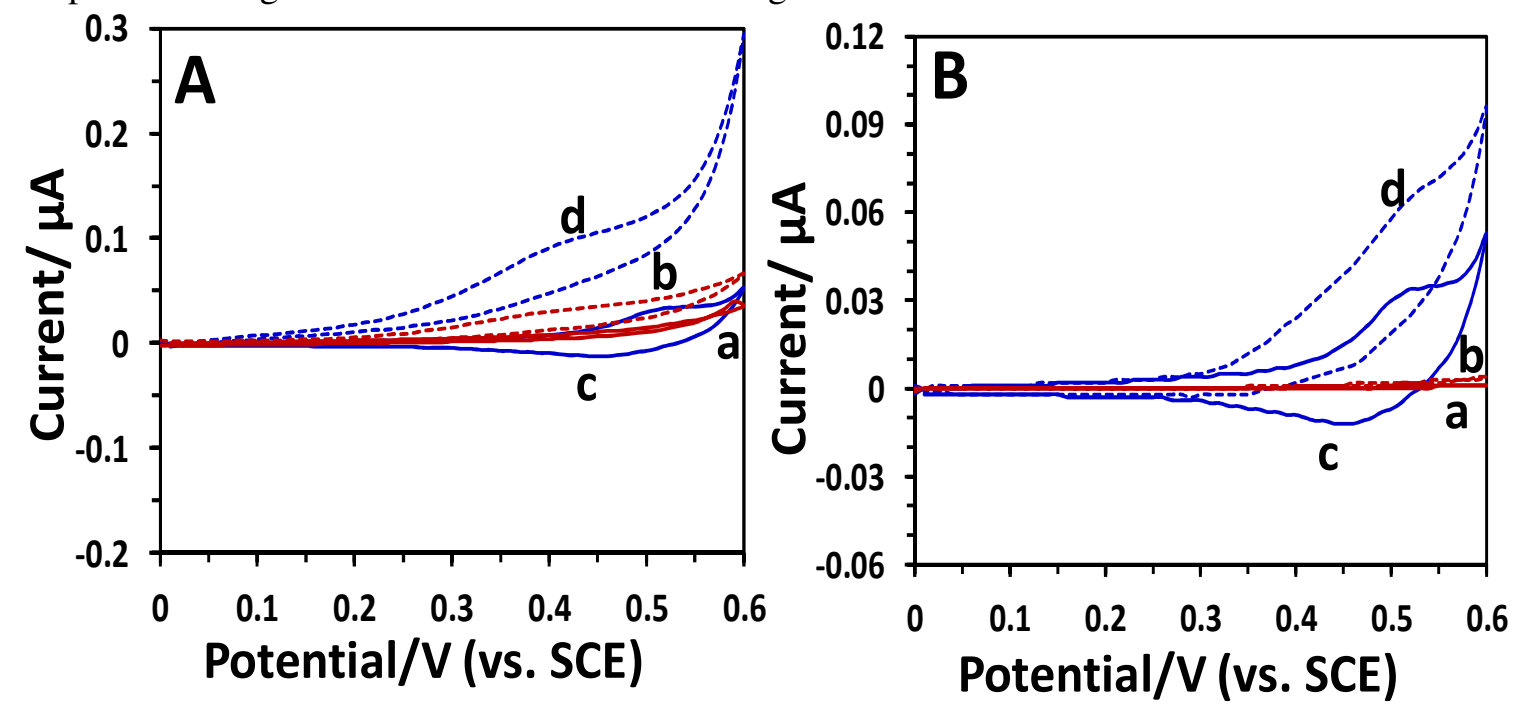

Fig.-2: (A) and (B) Cyclic Voltammograms in $0.1 \mathrm{M} \mathrm{NaOH}$ at a Scan Rate of $20 \mathrm{mV} \mathrm{s}^{-1}$. (a) Bare Electrode in the absence of Trp or Tyr, (b) Bare Electrode in the Presence of $890 \mu \mathrm{M}$ Trp or Tyr, (c) COSG-NC Modified Electrode

in the Absence of Trp or Tyr, (d) COSG-NC Modified Electrode in the Presence of $890 \mu \mathrm{M}$ Trp or Tyr.

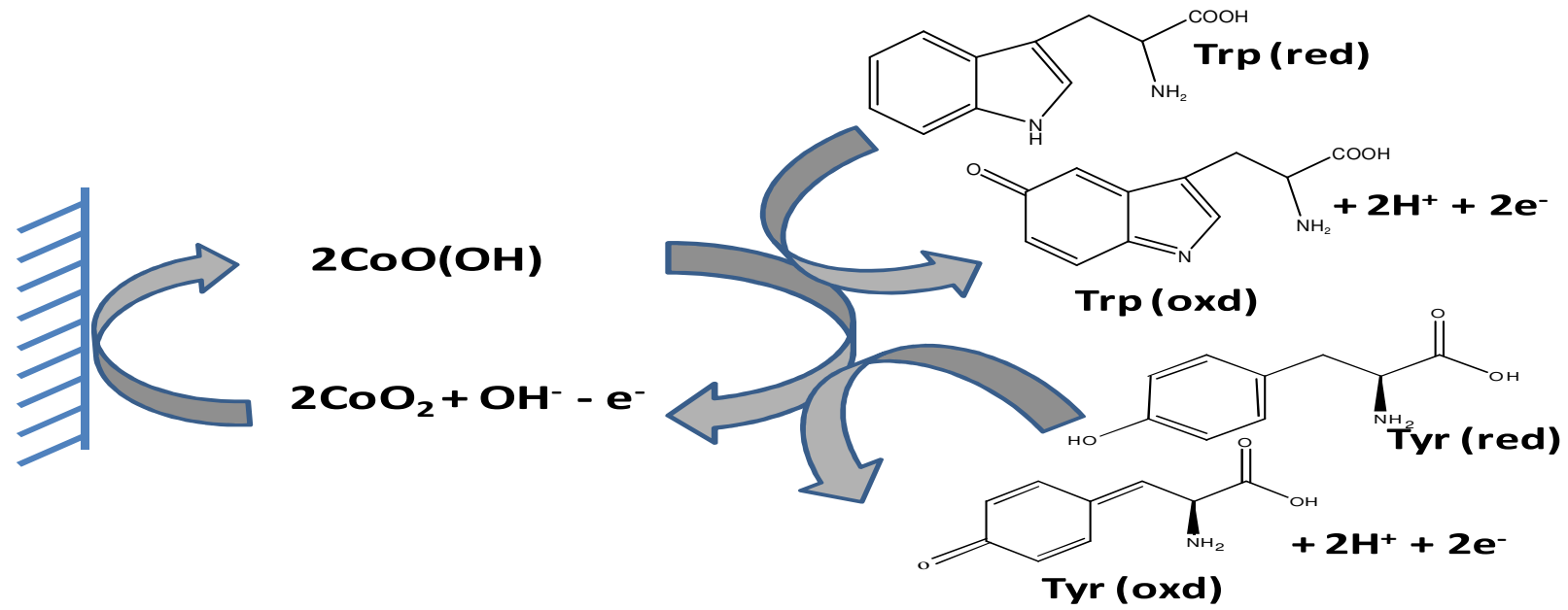

Scheme-1: Schematic representation of Electrocatalytic Oxidation Mechanism of Trp and Tyr at COSG-NC Modified Electrode

For Trp, on increasing step-wise the potential at $0.05 \mathrm{~V}$ intervals, the chronoamperometric response at the COSG-NC modified electrode starts at $0.30 \mathrm{~V}$ with subsequent increase in potential, the current rose 
sharply up to $0.45 \mathrm{~V}$ (Fig.-3A). However, in the Tyr determination with same COSG-NC modified electrode the oxidation current response starts at $0.40 \mathrm{~V}$, and the current rose sharply up to $0.55 \mathrm{~V}$ (Fig.-4A), which indicates good selectivity for the detection of Trp and Tyr. Hence, an applied potential of $0.45 \mathrm{~V}$ and $0.55 \mathrm{~V}$ for Trp and Tyr, respectively, were chosen for chronoamperometric studies. The corresponding calibration plots were plotted with catalytic current versus concentration of analytes and showed in Fig.-3B and 4B.
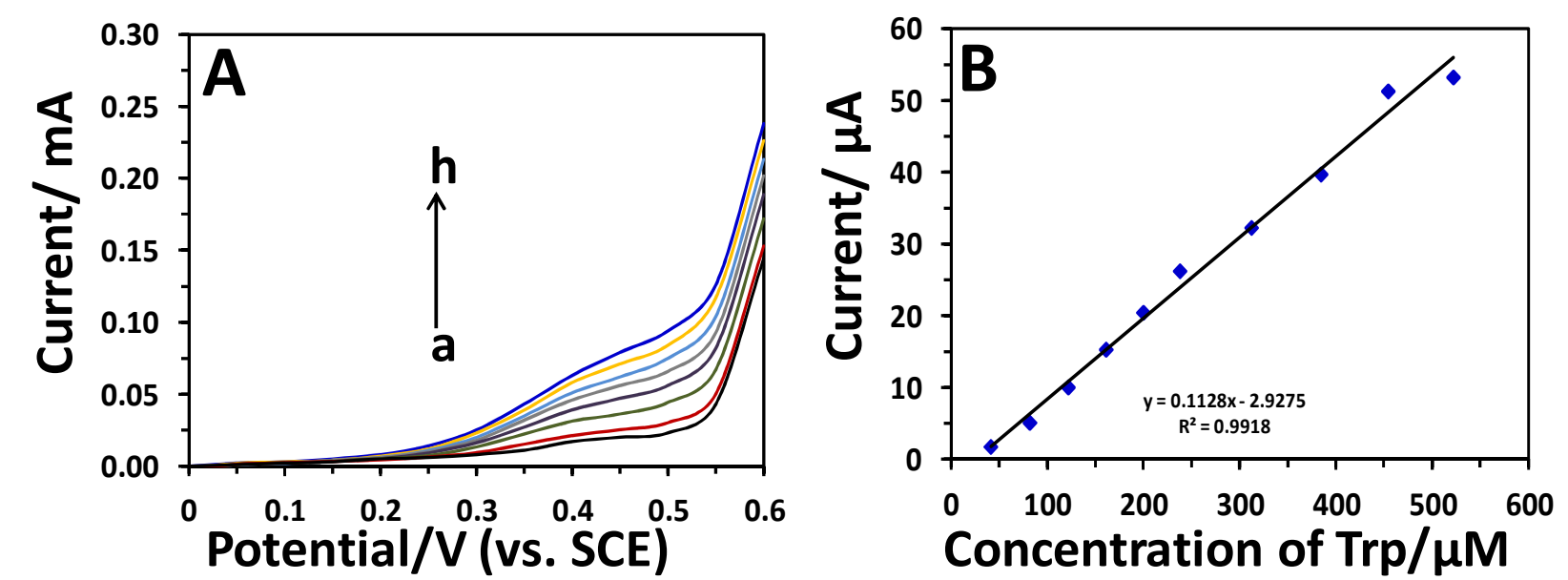

Fig.-3: (A) HDVs obtained with COSG-NC Modified Electrode in Different Concentrations from (a) $0 \mu \mathrm{M}$ to (h) $522 \mu \mathrm{M}$ of Trp. (B) Calibration Plot for Trp. Stirring Speed: $300 \mathrm{rpm}$, Electrolyte: $0.1 \mathrm{M} \mathrm{NaOH}$.
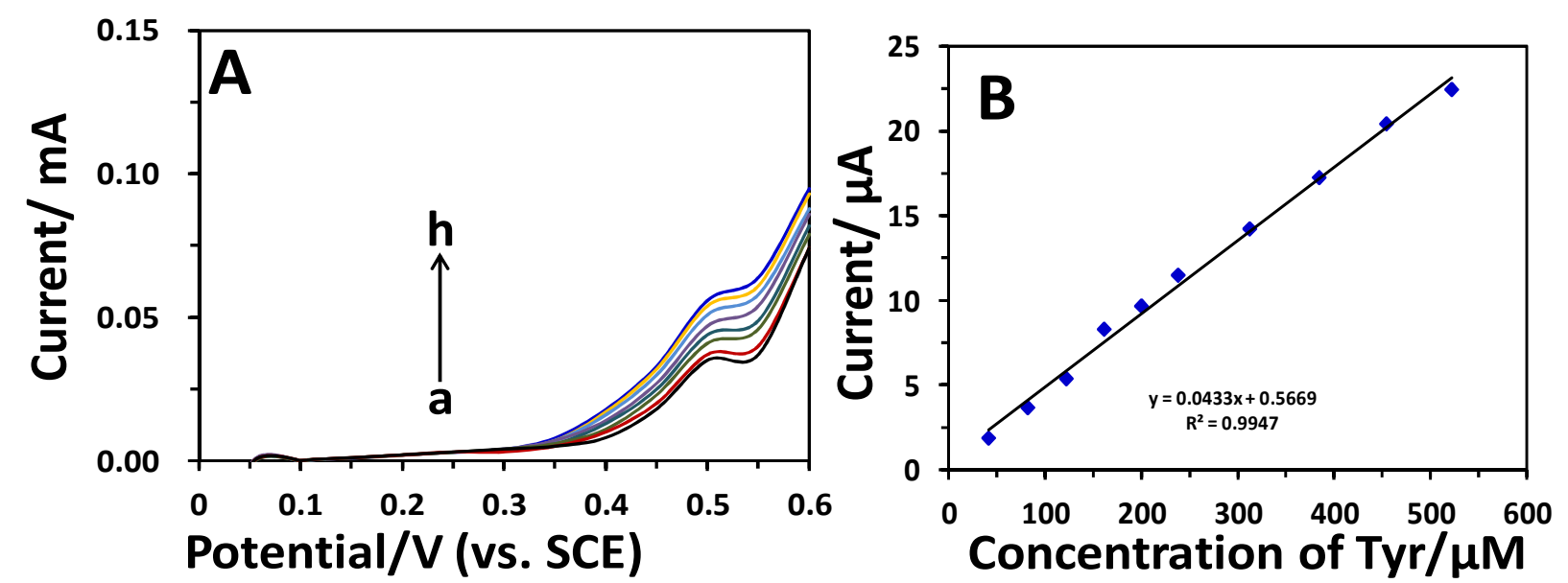

Fig.-4: HDVs obtained with COSG-NC Modified Electrode in Different Concentrations from (a) $0 \mu \mathrm{M}$ to (h) 522 $\mu \mathrm{M}$ of Tyr. (B) Calibration Plot for Tyr. Stirring Speed: $300 \mathrm{rpm}$, Supporting Electrolyte: $0.1 \mathrm{M} \mathrm{NaOH}$.

\section{Chronoamperometric Studies of Trp and Tyr}

To examine the applicability in the flow systems, COSG-NC modified electrode used as an amperometric sensor for the detection of Trp and Tyr in $0.1 \mathrm{M} \mathrm{NaOH}$ by chronoamperometry technique. From the HDV study, a potential of $0.45 \mathrm{~V}$ for $\operatorname{Trp}$ and $0.55 \mathrm{~V}$ for Tyr was fixed with respect to time for the chronoamperometric study. Figure-5A and 6A show a typical current-time response obtained at the COSG-NC modified electrode, which shows a stepwise increase in current response for successive increments of $5 \mathrm{mM}$ Trp and Tyr. Figure-5B and 6B illustrate the corresponding calibration plot showing a linear relationship between peak current and Trp and Tyr concentration in the range of $41 \mu \mathrm{M}$ to 380 $\mu \mathrm{M}$ with a correlation coefficient of 0.9935 and 0.9975 , respectively, for the part of the amperogram shown with a slope $0.032 \mu \mathrm{A} / \mu \mathrm{M}$ and $0.0306 \mu \mathrm{A} / \mu \mathrm{M}$. The response time for both substrates was found to be less than $3 \mathrm{~s}$. The performance of the COSG-NC modified electrode on repeated usage was also checked by carrying out the chronoamperometric experiment seven times with same concentration of Trp 
and $\operatorname{Tyr}(150 \mu \mathrm{M})$ and the R.S.D. was found to be 2.2 and $2.9 \%$, respectively. The results are indicative of the excellent reproducibility of the COSG-NC modified electrode for the detection of Trp and Tyr.
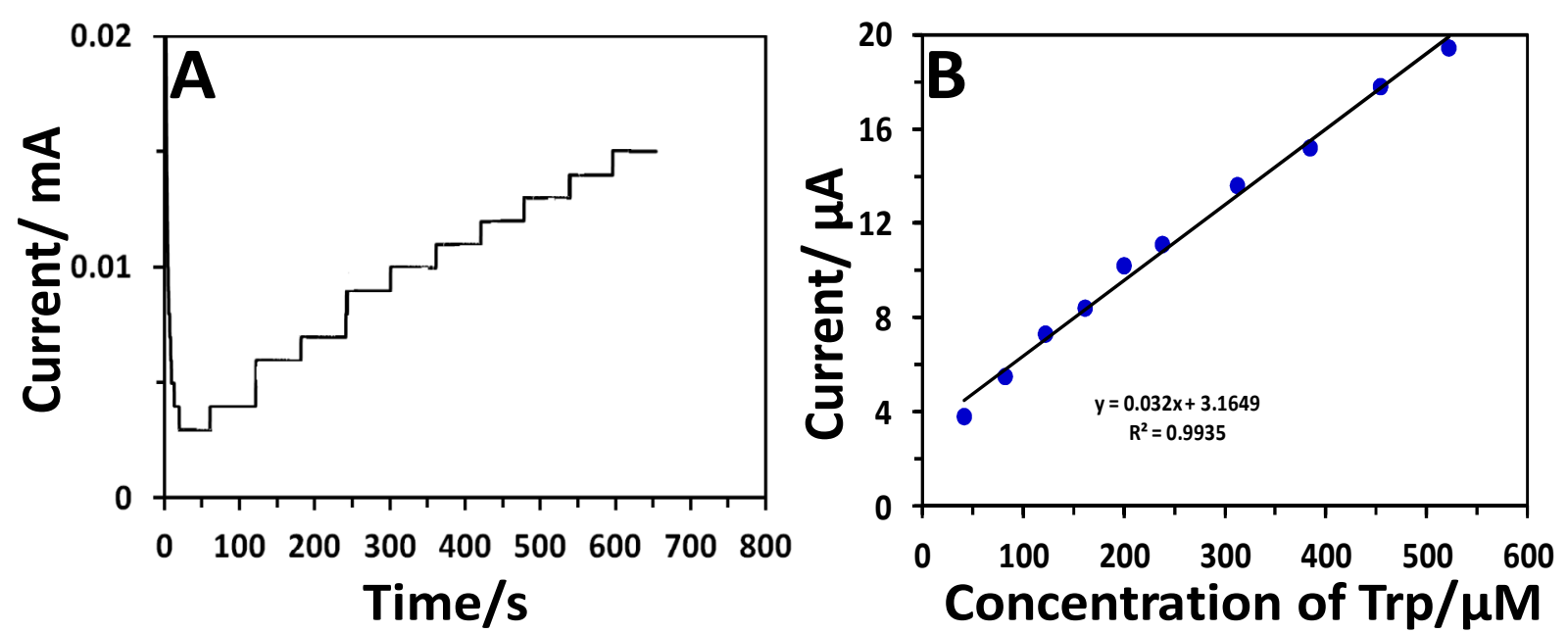

Fig.-5: (A) Chronomperometric responses of COSG-NC Modified Electrode to Consecutive Additions of $5 \mathrm{mM} \operatorname{Trp}$ in $0.1 \mathrm{M} \mathrm{NaOH}$ as Supporting Electrolyte at Fixed Potential of $+0.45 \mathrm{~V}$. (B) Calibration Plot for Variation of Current vs. Trp.
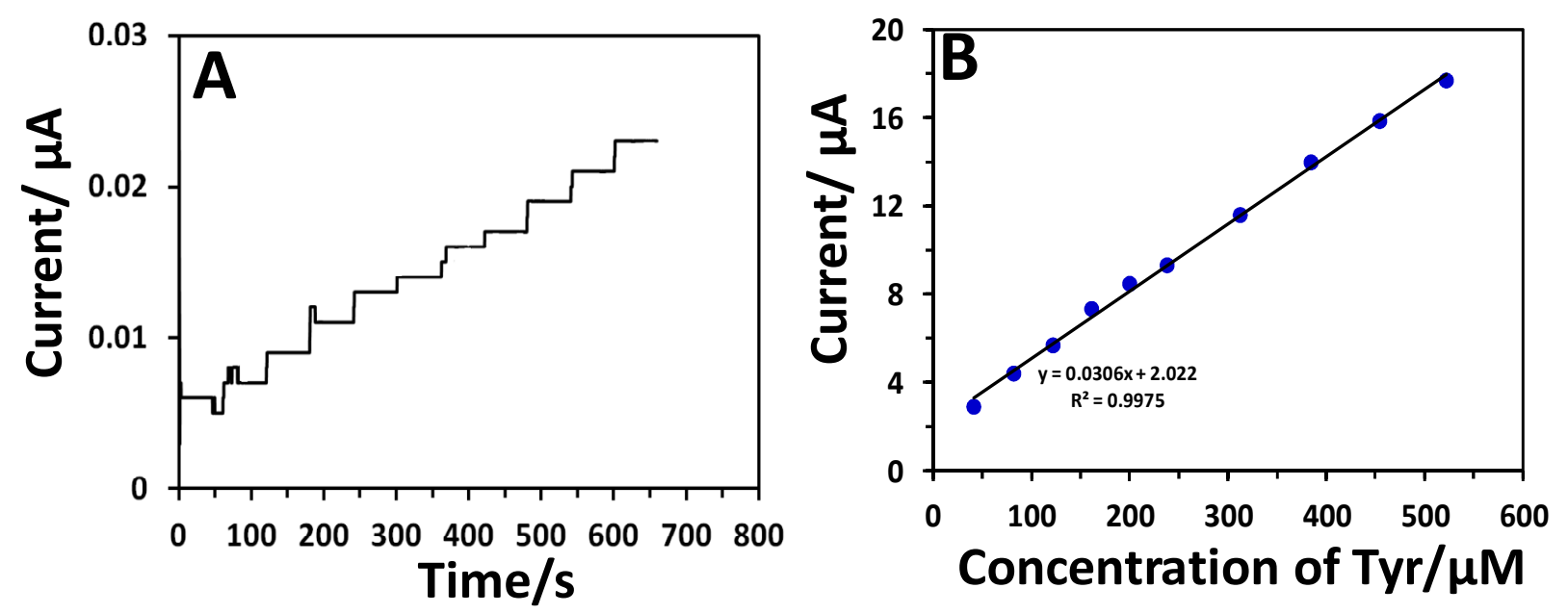

Fig.-6: (A) Chronoamperometric Studies of COSG-NC Modified Electrode to Consecutive Additions of $5 \mathrm{mM}$ Tyr in $0.1 \mathrm{M} \mathrm{NaOH}$ as Supporting Electrolyte at Fixed Potential of 0.55 V. (B) Calibration Plot for Variation of Current

Stability vs. Tyr.

The stability of the electrode modified with COSG-NC towards the electrocatalytic oxidation of $180 \mu \mathrm{M}$ Trp and Tyr were evaluated in long term. The proposed sensor retains $\sim 98.0 \%$ of its initial response even after 2 months of usage. The stable response of the COSG-NC modified electrode towards the electrooxidation of Trp and Tyr was evaluated for an extended period of six hours under stirred condition. The stable response of the COSG-NC modified electrode for the oxidation of $200 \mu \mathrm{M}$ Trp and Tyr recorded at an interval of 30 minutes and extended to 6 hours as demonstrated in Fig.-7, and it can be observed from the figure that the COSG-NC modified electrode exhibited nearly stable response signifying the efficient immobilization of the COSG-NC in the electrode surface and it is possible for the applicability in dynamic systems.

\section{Interference Studies}

Initially, the selectivity of COSG-NC modified electrode was analyzed individually towards the determination of Trp and Tyr in the existence of several common ions interferents for example $\mathrm{Na}^{+}, \mathrm{K}^{+}$, 
RASĀYAN J. Chem.

Vol. 12 | No. 3 |1166 - 1174| July - September | 2019

$\mathrm{NH}_{4}{ }^{+}, \mathrm{Mg}^{2+}, \mathrm{Ca}^{2+}, \mathrm{Cl}^{-}, \mathrm{F}^{-}, \mathrm{CO}_{3}{ }^{2-}$ and $\mathrm{SO}_{4}{ }^{2-}$ and various physiological interferents for example oxalate, urea, alanine, glycine and serine. In the presence of $5 \times 10^{-4} \mathrm{M}$ of $\mathrm{MgSO}_{4}, \mathrm{CaCl}_{2}, \mathrm{NaCl}, \mathrm{K}_{2} \mathrm{CO}_{3}, \mathrm{NH}_{4} \mathrm{Cl}$, urea, oxalate, glycine, alanine and serine, no significant changes in current response was observed for $1 \times$ $10^{-5} \mathrm{M}$ of Trp and Tyr, which indicates that the modified electrode is highly selective toward the determination of Trp and Tyr. Ascorbic acid (AA) can easily interfere in the detection of Trp and Tyr. AA can simply be eradicated by coating Nafion on the modified electrode surface. Trp and Tyr are coexisting in the pharmaceutical formulations, biological fluids and food processing. Various strategies have been developed for selective detection of Trp and Tyr. Since both substrates have analogous oxidation potentials at conventional solid electrodes, individual determination of these species is a problem due to the overlapped signals. Hence, the detection of Trp in the co-existence of Tyr with a good selectivity is a great challenge. In order to appraise the selectivity of the proposed sensor, the electrochemical behavior of the COSG-NC modified electrode towards the mixture of Trp and Tyr was observed at fixed applied potential of $0.45 \mathrm{~V}$ using amperometric method. In the case of Trp, its oxidation peak potential was lesser than Tyr oxidation potential based on our cyclic voltammetry and linear sweep voltammetry studies. Hence, the COSG-NC modified electrode determined selectively towards Trp and Tyr.

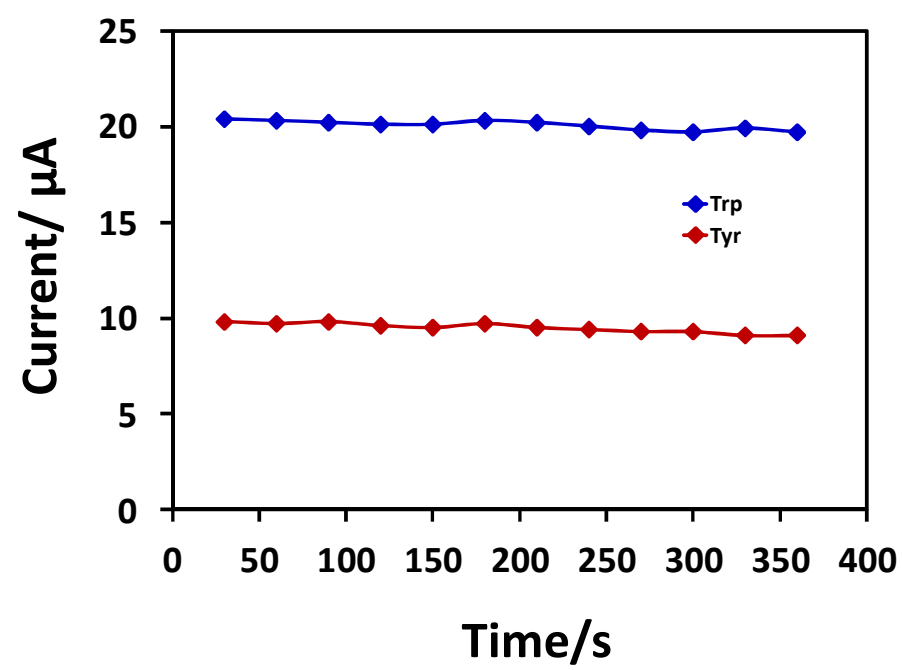

Fig.-7: Chronoamperometric response of COSG-NC Modified Graphite Electrode Towards the Sensing of $200 \mu \mathrm{M}$ Trp and Tyr Under Stirring Conditions for $6 \mathrm{~h}$.

\section{CONCLUSION}

In summary, graphene and cobalt oxide were successively sandwiched through self-assembly method and coated on a PIGE via simple drop and dry method. The resulted COSG-NC modified graphite electrode showed the outstanding electrocatalytic ability to the Trp and Tyr oxidation with extensive determination range, high sensitivity, low limit of detection, excellent reproducibility, selectivity, and good stability in long term, which can be ascribed to the combined effect of densely packed nanocomposites of twodimensional graphene and cobalt oxide. This work offers a practical approach to construct a selection of enzyme-free amperometric sensors.

\section{ACKNOWLEDGMENT}

This work was supported by the Defense Research and Development Organization (DRDO), New Delhi. The authors gratefully acknowledged VISTAS, Chennai, for providing infrastructure facilities.

\section{REFERENCES}

1. I. Gonzalez-Burgos, E. Olvera-Cortes, A.R. Del Angel-Meza and A. Feria-Velasco, Neurosci. Lett., 190, 143 (1995), DOI: 10.1016/0304-3940(95)11519-3

2. A. Carlsson and M. Lindqvist, Naunyn-Schmiedeberg's Arch. Pharmacol., 303, 157(1978), DOI: 10.1007/BF00508062 


\section{RASĀYAN J. Chem.}

Vol. 12 | No. 3 |1166 - 1174| July - September | 2019

3. A. Agazzi, F. De Ponti, R. De Giorgio, S.M. Candura, L. Anselmi, E. Cervio, A. Di Nucci and M. Tonini, Dig. Liver Dis., 35, 590 (2003), DOI: 10.1016/S1590-8658(03)00277-9

4. M.H. Fernstrom and J.D. Fernstrom, Life Sci., 57, 97 (1995), DOI: 10.1016/0024-3205(95)02026-F

5. Q. Xu and S.-F. Wang, Microchim. Acta, 151, 47 (2005), DOI: 10.1007/s00604-005-0408-6

6. J. Tashkhourian, M. Daneshi and S.F. Nami-Ana, Anal. Chim. Acta., 902, 89 (2016), DOI: 10.1016/j.aca.2015.10.037

7. M. Sa, L. Ying, T. Ai-Guo, X. Le-Dong and R. Ya-Ping, Clin. Chim. Acta, 413, 973(2012), DOI: 10.1016/j.cca.2012.02.019

8. A. Calvet, B. Li and A.G. Ryder, J. Pharm. Biomed. Anal., 71, 89(2012), DOI: $10.1016 / j . j p b a .2012 .08 .002$

9. P. Deng, Z. Xu and Y. Feng, Mater. Sci. Eng. C, Mater. Biol. Appl., 35, 54(2014), DOI: $10.1016 /$ j.msec.2013.10.019

10. A. Ozcan and Y. Sahin, Biosens. Bioelectron., 31, 26(2012), DOI: $10.1016 /$ j.bios.2011.09.048

11. X. Liu, L. Luo, Y. Ding, Z. Kang and D. Ye, Bioelectrochemistry, 86, 38(2012), DOI:10.1016/j.bioelechem.2012.01.008

12. S. Zhou, H. Wu, Y. Wu, H. Shi, X. Feng, H. Huang, J. Li and W. Song, Electrochim. Acta, 112, 90 (2013), DOI: 10.1016/j.electacta.2013.08.134

13. X. Liu, L. Luo, Y. Ding and D. Ye, Bioelectrochemistry, 82, 38(2011), DOI: $10.1016 /$ j.bioelechem.2011.05.001

14. C. Li, Y. Ya and G. Zhan, Colloids Surf. B Biointerfaces, 76, 340 (2010), DOI: 10.1016/j.colsurfb.2009.11.017

15. P. Prabhu, R.S. Babu and S.S. Narayanan, Colloids Surf. B Biointerfaces, 87, 103 (2011), DOI: 10.1016/j.colsurfb.2011.05.008

16. V. Sivasankar, E. Senthil Kumar, R.S. Babu, S. Raghu and R.A. Kalaivani, Rasyan J Chem., 10, 1232 (2017), DOI: $10.7324 /$ RJC.2017.1041749

17. R.S. Babu, P. Prabhu and S.S. Narayanan, RSC Adv., 4, 47497 (2014), DOI:10.1039/C4RA04507F

18. R.S. Babu, P. Prabhu and S.S. Narayanan, J. Nanosci. Nanotechol., 16, 8711 (2016), DOI:10.1166/jnn.2016.11709

19. R.S. Babu, P. Prabhu and S.S. Narayanan, Talanta, 110, $135 \quad$ (2013), DOI:10.1016/j.talanta.2013.02.025

20. S. Elhag, Z.H. Ibupoto, X. Liu, O. Nur and M. Willander, Sens. Actuators B, 203, 543 (2014), DOI: 10.1016/j.snb.2014.07.028

21. C.-W. Kung, C.-Y. Lin, Y.-H. Lai, R. Vittal and K.-C. Ho, Biosen. Bioelectron., 27, 125 (2011), DOI: $10.1016 /$ j.bios.2011.06.033

22. R. Vivekananth, R.S. Babu, K. Prasanna, C.W. Lee and R.A. Kalaivani, J. Mater. Sci. Mater. Electron., 29, 6763 (2018), DOI: 10.1007/s10854-018-8662-7

23. Y. Ding, Y. Wang, L. Su, M. Bellagamba, H. Zhang and Y. Lei, Biosen. Bioelectron., 26, 542 (2010), DOI: $10.1016 /$ j.bios.2010.07.050

24. H. Yin, J. Zhu, J. Chen, J. Gong and Q. Nie, J. Mater. Sci. Mater. Electron., 29, 17305 (2018), DOI: 10.1007/s10854-018-9825-2

25. L. Wang, Y. Zhang, Y. Xie, J. Yu, H. Yang, L. Miao and Y. Song, Appl. Surf. Sci., 402, 47 (2017), DOI: $10.1016 /$ j.apsusc.2017.01.062

26. L. Kang, D. He, L. Bie and P. Jiang, Sens. Actuators B, 220, 888 (2015), DOI: 10.1016/j.snb.2015.06.015

27. Z.J. Wang, X.Z. Zhou, J. Zhang, F. Boey and H. Zhang, J. Phys. Chem. C, 113, 14071 (2009), DOI: doi/10.1021/jp906348x

28. K.S. Novoselov, A.K. Geim, S.V. Morozov, D. Jiang, Y. Zhang, S.V. Dubonos, I.V. Grigorieva and A.A. Firsov, Science, 306, 666 (2004), DOI: 10.1126/science.1102896

29. W.J. Hong, Y.X. Xu, G. W. Lu, C. Li and G.Q. Shi, Electrochem. Commun., 10, 1555 (2008), DOI: 10.1016/j.elecom.2008.08.007 
RASĀYAN J. Chem.

Vol. 12 | No. 3 |1166 - 1174| July - September | 2019

30. H. Gómez, M.K. Ram, F. Alvi, P. Villalba, E. Stefanakos and A. Kumar, J. Power Sources, 196, 4102 (2011), DOI: 10.1016/j.jpowsour.2010.11.002

31. F.N. Xia, D.B. Farmer, Y.M. Lin and P. Avouris, Nano Lett., 10, 715 (2010), DOI: $10.1021 / \mathrm{n} 19039636$

32. G.H. Lu, L.E. Ocola and J.H. Chen, Nanotechnology, 20, 445 (2009), DOI: 10.1088/09574484/20/44/445502

33. C.S. Shan, H.F. Yang, D.X. Han, Q.X. Zhang, A. Ivaska and L. Niu, Biosens. Bioelectron., 25, 1070 (2010), DOI: 10.1016/j.bios.2009.09.024

34. S.J.R. Prabakar, R.S. Babu, M. Oh, M.S. Lah, S.C. Han, J. Jeong and M. Pyo, J. Power Sources, 272, 1037 (2014), DOI: 10.1016/j.jpowsour.2014.09.044

35. R.S. Babu, P. Prabhu and S.S. Narayanan, J. Solid State Electrochem., 20, 1575 (2016), DOI: 10.1007/s10008-016-3161-3

[RJC-5045/2019] 